\title{
IN VITRO ANTIOXIDANT ACTIVITY AND PUNICALAGIN CONTENT QUANTIFICATION OF POMEGRANATE PEEL OBTAINED AS AGRO-WASTE AFTER JUICE EXTRACTION
}

\author{
Anees Ahmed Khalil ${ }^{1, *}$, Moazzam Rafiq Khan², Muhammad Asim Shabbir² and \\ Khalil-ur-Rahman ${ }^{3}$
}

\author{
${ }^{1}$ University Institute of Diet and Nutritional Sciences, Faculty of Allied Health Sciences, The University of Lahore- \\ Lahore; ${ }^{2}$ National Institute of Food Science and Technology, Faculty of Food, Nutrition and Home Sciences, \\ University of Agriculture Faisalabad, Pakistan; ${ }^{3}$ Department of Biochemistry, Faculty of Sciences, University of \\ Agriculture Faisalabad, Pakistan. \\ "Corresponding author's e-mail: aneesahmedkhalil@gmail.com
}

\begin{abstract}
The aim of current investigation was to assess total phenolics (TPC), total flavonoids (TFC) and punicalagin content (PC) of pomegranate peel extracts (PPEs) for its utilization as functional ingredient in food processing industry. Antioxidant rich fractions were extracted from Punica granatum L. (pomegranate) peel using methanol, ethanol and ethyl acetate. Furthermore, the extracts were characterized to assess their antioxidant potential using in vitro DPPH assay model. Methanolic extracts inhibited $78.23 \%$ free radicals; however ethyl acetate extracts showed least antioxidant activity. Maximum phenolic and flavonoid contents were extracted in methanolic extracts that were documented as $289.40 \pm 12.75 \mathrm{mg} / \mathrm{g} \mathrm{GAE}$ (Gallic acid equivalents) and $58.63 \pm 3.41 \mathrm{mg} / \mathrm{g} \mathrm{RE}$ (Rutin equivalents), respectively, whereas punicalagin $(110.00 \pm 5.10 \mathrm{mg} / \mathrm{g}) \mathrm{was}$ the major ellagitannin detected and quantified. Significant correlation $(r=0.981,0.958 ; N=3)$ was observed between total phenolics, total flavonoids and antioxidant activity of respective extracts. Correlation analysis $(r=0.982 ; N=3)$ suggested that punicalagin might be the reason for potent antioxidant activity of PPEs. Conclusively, the outcomes of this study could serve as baseline information for fruits and vegetable processors in formulation of nutraceutical and therapeutic designer foods.
\end{abstract}

Keywords: Phytogenic compounds, antioxidants, punicalagin, HPLC, nutraceuticals.

\section{INTRODUCTION}

Numerous individuals suffering from metabolic syndromes like diabetes, arthritis, cardiovascular diseases (CVDs) and cancer are mainly due to age factor or genetic make-up (Ignarro et al., 2007). On the other hand, sedentary lifestyle including poor dietary habits and inadequate physical activity, alongside impact of environmental pollution may also be the reason for onset of these disorders. Furthermore, oxidative stress conditions produce reactive oxygen species (ROS) that interact with cell structure of human body causing deleterious diseases including oncogenic and neuro-degenerative ailments (Ferrari, 2007). Fruits and vegetables containing naturally occurring antioxidants such as hydrolysable tannins (punicalagin and punicalin), phenolics (gallic acid and ellagic acid) flavonoids (catechin and rutin), are undoubtedly significant tool in prevention of these disorders (Han et al., 2008).

During the last few decades, food processors are using synthetic antioxidants for the prevention of lipid peroxidation; a key reason for quality deterioration on food materials. They are useful in reduction of oxidation but due to their chemical nature and recent exploration regarding health risks associated with their use in the food products, consumer preferences have shifted towards use of naturally occurring antioxidants in fruits and vegetables. Therefore, nutritionists are emphasizing on identification and quantification of different fruit and vegetable based bioactive compounds to ensure ultimate human health (Balasundram et al., 2006). So far, significant literature is available on antioxidant potential of different plant based phytochemicals. In spite of this, agrowastes of fruit and vegetable processing industries comprise cache of phytogenic compounds which could be explored to enhance human health status and nutritional quality of food (Reddy et al., 2007). The pomegranate (Punica granatum L.) fruits are rich source of fiber and polyphenolics including ellagitannins, anthocyanins and gallic acids (Negi et al., 2003; Noda et al., 2002). Pomegranate fruit based antioxidants are useful in reducing progression of various chronic diseases. Currently, scientists are focusing on exploiting naturally occurring phytochemicals present in structural matrix (ViudaMartos et al., 2011).

Purposely, the present study was intended to enhance the knowledge regarding pomegranate peel polyphenols extracted by using three different solvents (methanol, ethanol and ethyl acetate) based on quantification of punicalagin, a bioactive compound responsible for its potent antioxidant activity, through HPLC-UV. Additionally, free radical 
scavenging antioxidant activity was evaluated by performing DPPH assay. To exploit pomegranate peel's nutraceutical potential, total phenolics and flavonoids were determined in this study.

\section{MATERIALS AND METHODS}

Chemicals and reagents: 2,2-diphenyl-1-picrylhydrazyl (DPPH), Folin-Ciocalteu reagent, gallic acid, rutin, Trifluoroacetic acid(TFA), tri-chloroacetic acid (TCA), aluminium chloride $\left(\mathrm{AlCl}_{3}\right)$ were purchased from Sigma. Sodium nitrite $\left(\mathrm{NaNO}_{2}\right)$, sodium hydroxide $(\mathrm{NaOH})$ and sodium carbonate $\left(\mathrm{Na}_{2} \mathrm{CO}_{3}\right)$ were procured from Merck. HPLC grade punicalagin and methanol were also supplied by Sigma Chemical Company (Germany).

Procurement of raw material and sample preparation: Mature and healthy pomegranate fruits without any visible bruising were procured from the local market. Respective peels were obtained by separately peeling each pomegranate manually. Pomegranate peels were then dried in cabinet dryer at $60^{\circ} \mathrm{C}$ for 24 hours. The dried peels were ground to fine powder and passed through a No. 30 mesh sieve. Finally, prepared powders were stored in plastic jars at ambient temperature till further analysis.

Extraction of polyphenols: The antioxidant extract of pomegranate peel powder samples were obtained by treating them with different solvents such as methanol (50\%), ethanol $(50 \%)$ and ethyl acetate $(50 \%)$, respectively. For this purpose, prepared mixtures were subjected to orbital/mechanical shaker for seven hours trailed by centrifugation for 15 minutes at 12,000 rpm (Viuda-Martos et al., 2011). Obtained resultant pomegranate peel raw extracts were vacuum-filtered (Rusak et al., 2008).

Determination of total phenolic contents (TPC): Total phenolic contents in each pomegranate peel extract (PPE) were assessed by adopting the protocol of Singleton et al. (1999). Accordingly, extract measuring $125 \mu \mathrm{L}$ was added in $500 \mu \mathrm{L}$ distilled $\mathrm{H}_{2} \mathrm{O}$ along with addition of Folin-Ciocalteu reagent @ $125 \mu \mathrm{L}$ and this mixture was held-up for 5 minutes. After standing time, $1.25 \mathrm{~mL}$ of $7 \% \mathrm{Na}_{2} \mathrm{CO}_{3}$ solution was added in the mixture. Final volume was maintained up to 3 $\mathrm{mL}$ by adding distilled water. After standing time of 90 minutes, the absorbance was measured using UV-Visible Spectro-photometer (CECIL, CE7200) at $765 \mathrm{~nm}$. Total phenolics were determined and expressed as $\mathrm{mg}$ Gallic acid equivalent (GAE)/g.

Determination of total flavonoid contents (TFC): Total flavonoid contents (TFC) were determined by aluminum chloride calorimetric method (Chang et al., 2002). For this purpose, $50 \mu \mathrm{L}$ of pomegranate peel extract were raised up to $1 \mathrm{~mL}$ by adding methanol, mixed with $4 \mathrm{~mL}$ of distilled water followed by addition of $0.3 \mathrm{~mL}$ of $5 \% \mathrm{NaNO}_{2}$ and $0.3 \mathrm{~mL}$ of $10 \% \mathrm{AlCl}_{3}$ after $5 \mathrm{~min}$ of incubation. Then resultant mixture was placed for further 6 minutes. Afterwards, $2 \mathrm{~mL}$ of $1 \mathrm{M}$
$\mathrm{NaOH}$ solution was added and volume was raised up to 10 $\mathrm{mL}$ by adding distilled water. For all the samples the absorbance was measured at $510 \mathrm{~nm}$ by using UV/vis Spectro-photometer (CECIL CE7200). All findings were stated as mg rutin equivalents (RE)/g.

Measurement of in vitro antioxidant potential (DPPH): The antioxidant capacity of resultant peel extracts in all three solvents was calculated in terms of free radical scavenging potential by using DPPH-assay (Brand-Williams et al., 1995). Briefly, each peel extract $(4 \mathrm{~mL})$ were placed in the cuvette followed by the addition of $1 \mathrm{~mL}$ of DPPH methanolic solution. The resultant mixture was allowed to stand for 30 minutes at $25^{\circ} \mathrm{C}$. The absorbance of resultant mixture was noticed at $520 \mathrm{~nm}$ through UV-Vis Spectro-photometer (CECIL CE7200). Percent inhibition was measured using following formula:

$$
\text { Reduction in absorbance }(\%)=\frac{\mathrm{AB}(\mathrm{s})-\mathrm{AB}(\mathrm{e})}{\mathrm{AB}(\mathrm{s})} \times 100
$$

$\mathrm{AB}(\mathrm{s})=$ absorbance of blank sample $(\mathrm{t}=0 \mathrm{~min})$

$\mathrm{AB}(\mathrm{e})=$ absorbance of tested extract solution $(\mathrm{t}=30 \mathrm{~min})$

Punicalagin quantification (HPLC-UV): Quantification of punicalagin was performed using HPLC (Perkin-Elmer, Series 200, USA) having $\mathrm{C}_{18}$ column (dimensions: $250 \mathrm{~mm} \mathrm{x}$ $4.6 \mathrm{~mm}$, particle size: $5 \mu \mathrm{m}$ ). A sample of $10 \times 10^{-6} \mathrm{~L}$ was taken up by auto sampler and $30^{\circ} \mathrm{C}$ temperature of column was ensured throughout the analytical process. Throughout analysis; mobile phase comprised of Methanol (eluent-A) and $0.1 \%$ ( $\mathrm{vol} / \mathrm{vol}$ ) Trifluoroacetic acid in HPLC grade water (eluent-B). Chromatographic conditions (Gradient): 0-10 minutes, 5-20\% A in B; 10-20 minutes, 20-40\% A in B; 2026 minutes, $70 \% \mathrm{~A}$ in $\mathrm{B}$. These protocols were trailed by reequilibrium for 10 minutes. The flow rate was adjusted at 1 $\mathrm{mL} \min ^{-1}$ and punicalagin was quantified at $378 \mathrm{~nm}$ wavelength using UV-Vis detector (Lu et al., 2011).

Statistical analysis: Data was statistically analyzed using one-way ANOVA and significant difference $(P<0.05)$ was measured by Tukey's HSD test. All results were expressed in triplicate as means \pm SD.

\section{RESULTS AND DISCUSSION}

It is vitally important to understand phenolic composition and potent antioxidant properties of fruit extracts before their ultimate utilization in food processing industry. Different analytical tools including spectrophotometer and HPLC-UV were used to generate a database which could authenticate the use of pomegranate peel as an economical and easily available fruit waste for preparation of polyphenolic rich extracts having strong antioxidant activity and nutraceutical potential. Total phenolics and flavonoids in pomegranate peel extracts: Plants based phenolics generally act as therapeutic agents, for instance prevention from cancers, antioxidant agents and potent antibacterial (Adnan et al., 2011). Results of present exploration for total phenolic and flavonoid 
contents of pomegranate peel extracts (PPEs) are illustrated in Table 1. The total phenolic contents (TPC) of PPEs ranged from $236.12-289.40 \mathrm{mg} / \mathrm{g}$ GAE in different solvents with methanol exhibiting the highest content and ethyl acetate showing the least. The statistical analysis revealed that total phenolics in PPEs were significantly $(P<0.05)$ affected by the type and nature of the solvent. The results illuminated that both polarity difference and affinity of pomegranate peel polyphenols towards used solvents strongly influenced phenolic composition. Means for total phenols in methanolic PPEs $(289.40 \pm 12.75 \mathrm{mg} / \mathrm{g}$ GAE) was 13.05 and $18.41 \%$ higher as compared to ethanolic PPEs $(251.62 \pm 10.82 \mathrm{mg} / \mathrm{g}$ GAE) and ethyl acetate PPEs (236.12 $\pm 8.38 \mathrm{mg} / \mathrm{g}$ GAE). Likewise, average amount of total flavonoid contents (TFC) in different PPEs exposed that the maximum value was noticed in methanol $(58.63 \pm 3.41 \mathrm{mg} / \mathrm{g} \mathrm{RE})$ followed by ethanol $(55.26 \pm 2.25 \mathrm{mg} / \mathrm{g} \mathrm{RE})$ and minimum output $(46.71 \pm 3.69 \mathrm{mg} / \mathrm{g} \mathrm{RE})$ was recorded in ethyl acetate extract.

Table 1. Total phenolic contents of pomegranate peel extracts using different solvents.

\begin{tabular}{lcc}
\hline Solvents & $\begin{array}{c}\text { Total Phenolic } \\
\text { Contents* }^{*}\end{array}$ & $\begin{array}{c}\text { Total Flavonoid } \\
\text { Contents** }^{* *}\end{array}$ \\
\hline Methanol & $289.40^{\mathrm{a}} \pm 7.36$ & $58.63^{\mathrm{a}} \pm 3.41$ \\
Ethanol & $251.62^{\mathrm{b}} \pm 6.25$ & $55.26^{\mathrm{a}} \pm 2.25$ \\
Ethyl Acetate & $236.12^{\mathrm{b}} \pm 4.84$ & $46.71^{\mathrm{b}} \pm 3.69$ \\
\hline
\end{tabular}

Each value is expressed as means $\pm \mathrm{SD}$; ${ }^{*}$ Expressed as $\mathrm{mg} / \mathrm{g}$ Gallic acid equivalent (GAE); **Expressed as $\mathrm{mg} / \mathrm{g}$ Rutin equivalent (RE)

The mean concentration of total flavonoids extracted in methanol was almost 5.74 and $20.33 \%$ more in comparison to ethanol and ethyl acetate (Fig. 1a and 1b). Similarly, Singh et al. (2002) concluded that methanolic extracts have maximum phenolics yield, mainly due to the ascribed polarity differences among the type of solvent and nature of polyphenolic compounds to be extracted. These polyphenolic molecules are mainly known for their free radical scavenging properties that ultimately inhibit lipid peroxidation (Noda $e t$ al., 2002).
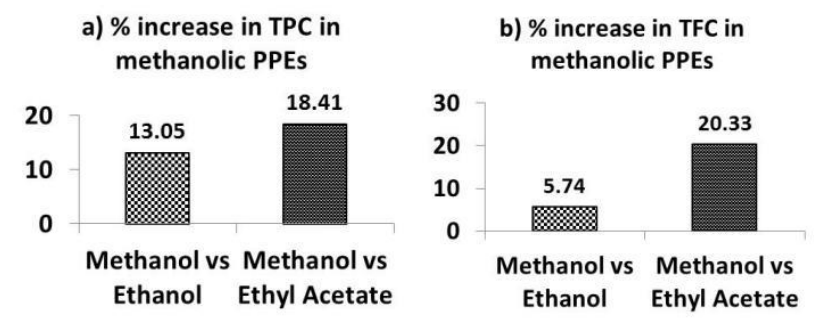

Figure 1. Extraction efficiency of methanol over ethanol and ethyl acetate extracts. The results are presented as mean \pm SD for PPEs using three solvents.

DPPH free radical scavenging activity of the different PPEs: To assess in vitro antioxidant potential of PPEs performed DPPH assay based on measuring the discoloration of DPPH radical at $520 \mathrm{~nm}$ after its reaction with antioxidant compound present in prepared extracts. Up till now, DPPHassay is the most commonly practiced process to evaluate the anti-oxidant capacity of respective extracts mainly due to its short run time, simplicity \& stability during experimental analysis. Primarily, the antioxidative activity of experimented samples is calculated depending upon their potential to reduce DPPH'. Mainly, by transferring $\mathrm{H}$-atom which could also be verified through the loss of dark violet color of the tested solution using spectrophotometer (Brand-Williams et al., 1995).

All PPEs, especially methanolic PPEs, were most effective in demonstrating strong free radical scavenging ability (DPPH). Significant correlations (Pearson's correlation coefficients $r=0.981,0.958 ; N=3$ ) were noticed among total phenolics, total flavonoids and free radical scavenging activity of PPEs. The results infer that pomegranate peel antioxidative extracts might be helpful in retarding food deterioration caused due to free radicals production. It is evident from Fig. 2 that methanolic extracts demonstrated maximum free radical quenching effect with highest antioxidant activity as compared to ethanolic and ethyl acetate extracts. The average values for solvents revealed that methanolic extracts significantly $(\mathrm{p}<0.05)$ scavenged maximum DPPH free radicals (78.23\%) followed by ethanol $(70.38 \%)$ and ethyl acetate $(63.36 \%)$.

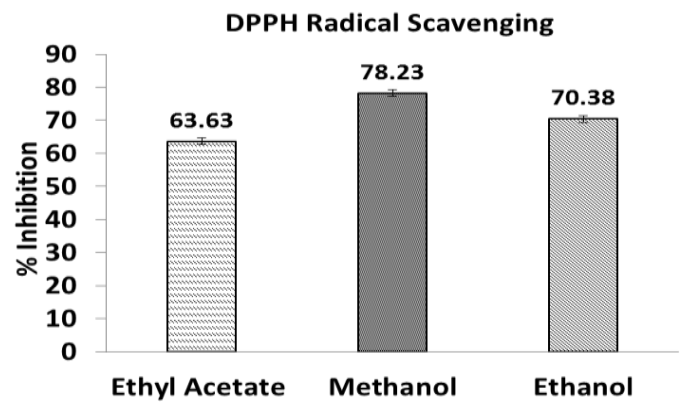

Figure 2. Comparison of the DPPH radical scavenging properties of different phytogenic extracts from Punica grantum L. peel. The results are presented as mean \pm SD for PPEs using three solvents.

High total phenolic contents in phytogenic extracts were generally associated with high free radical scavenging activity. Furthermore, phenolic complexes showed more affinity towards polar solvents as indicated elsewhere (Orak et al., 2012). Therefore, antioxidative potential of pomegranate peel was observed maximum in methanolic extracts, mostly due to the presence of high amount of polyphenols, such phenolics and flavonoids, having hydrogen donating ability helpful in scavenging and quenching free radicals (Middha et al., 2013). Earlier Brand-Williams et al. (1995) was of opinion that scavenging potential of phenolic 
compounds is associated to their structural properties and resultant antioxidative properties are directly correlated to amount of existing OH-groups. All above mentioned activities might be associated to various poly-phenolic complexes present in peel of pomegranate peel, including $\alpha$, $\beta$ isomers of punicalagin, ellagic acid (EA) derivatives \& flavonoids (rutin, catechin and quercetin). In Vitro, these compounds are recognized due to their free radical scavenging and lipid-oxidation inhibitory properties (Gil et al., 2000).

HPLC quantification of punicalagin content: Punicalagin content in pomegranate peel extracts were quantified and compared with pure standard of punicalagin thorough HPLCUV system (Fig. 3). Acquired results displayed in Table 2 were expressed as $\mathrm{mg} / \mathrm{g}$ punicalagin content and were substantially affected by the type of solvent.

Table 2. Total Punicalagin content in different extracts of pomegranate peel.

\begin{tabular}{lc}
\hline Solvents & Punicalagin Content $*$ \\
\hline Methanol & $110.00^{\mathrm{a}} \pm 5.10$ \\
Ethanol & $96.50^{\mathrm{ab}} \pm 3.46$ \\
Ethyl Acetate & $88.74^{\mathrm{b}} \pm 4.25$ \\
\hline Ea
\end{tabular}

Each value is expressed as means $\pm \mathrm{SD}$; *Expressed as $\mathrm{mg} / \mathrm{g}$.

Methanol was able to extract major portion of punicalagin present in pomegranate peel i.e. $110.00 \pm 5.10 \mathrm{mg} / \mathrm{g}$ dry weight of extract. That was trailed by ethanolic and ethyl acetate extracts with $96.50 \pm 3.46$ and $88.74 \pm 4.25 \mathrm{mg} / \mathrm{g}$ dry weight extract, correspondingly. Pearson's correlation coefficients $(r=0.982 ; N=3)$ showed significant correlation between total punicalagin content and free radical scavenging activity of PPEs. The existing outcomes are in harmony with the results of Lu et al. (2008) who revealed that punicalagin content in pomegranate husk ranged from $44.90-121.50 \mathrm{mg} / \mathrm{g}$ in fourteen different varieties. Punicalagin, most abundantly present in pomegranate peel is predominantly responsible for its potent antioxidant activity (Cam and Hisil, 2010).

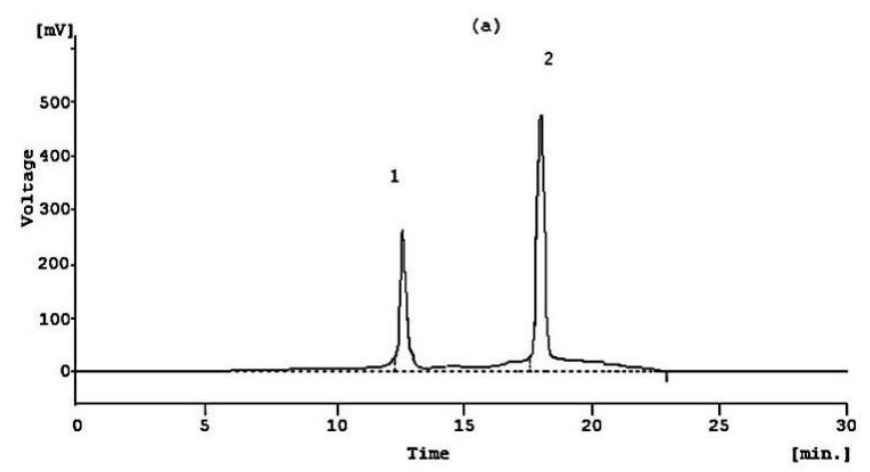

Figure 3. HPLC chromatograms of PPEs (a) punicalagin standard: $\alpha$-punicalagin(1) and $\beta$ punicalagin(2).
Conclusion: The outcomes of current study provide evidence that extracts prepared from different solvents have variable antioxidant activity depending upon their polarity and affinity towards phytogenic molecules present in pomegranate peel. Total phenolic and flavonoid contents were highest in methanolic extracts (289.40 mg/g GAE and $58.63 \mathrm{mg} / \mathrm{g} \mathrm{RE}$ ) as compared to other used solvents making it the most promising solvent in terms of polyphenolic extraction. Momentous correlation among phenolics and antioxidant activity was noticed in this study. Pomegranate peel extracts exhibited significantly strong antioxidant activity when determined using DPPH assay, reflecting its therapeutic role. Methanolic extract demonstrated highest free radical scavenging potential i.e. $78.23 \%$ which differed significantly $(p<0.05)$ from ethanolic $(70.38 \%)$ and ethyl acetate extract $(63.36 \%)$. Punicalagin $(110.00 \pm 5.10 \mathrm{mg} / \mathrm{g})$ was documented to be predominant bioactive compound in methanolic extracts of pomegranate peel. In a nutshell, the study illustrated the therapeutic prospective \& possible use of pomegranate peel as a cache of natural antioxidant.

Acknowledgement: The authors are thankful to Higher Education Commission (HEC), Pakistan for supporting this research. Corresponding author is obliged to co-authors for their keen interest and guidance.

\section{REFERENCES:}

Adnan, L., A. Osman and A. Abdul Hamid. 2011. Antioxidant activity of different extracts of Red Pitaya (Hylocereus polyrhizus) seed. Int. J. Food Prop. 14:1171-1181.

Balasundram, N., K. Sundram and S. Samman. 2006. Phenolic compounds in plants and agri-industrial byproducts: Antioxidant activity, occurrence, and potential uses. Food Chem. 99:191-203.

Brand-Williams, W., M.E. Cuvelier and C. Berset. 1995. Use of a free radical method to evaluate antioxidant activity. Lebensm. Wiss. Technol. 28:25-30.

Çam, M. and Y. Hisil. 2010. Pressurized water extraction of polyphenols from pomegranate peels. Food Chem. 123:878-885.

Chang, C., M. Yang, H. Wen and J. Chern. 2002. Estimation of total flavonoid content in propolis by two complementary colorimetric methods J. Food Drug Anal. 10:178-182.

Ferrari, C.K.B. 2007. Functional foods and physical activities in health promotion of aging people. Maturitas 58:327339.

Gil, M.I., F.A. Tomàs-Barberàn, B. Hess-Pierce, D.M. Holcroft and A.A. Kader. 2000. Antioxidant activity of pomegranate juice and its relationship with phenolic composition and processing. J. Agric. Food Chem. 48:4581-4589. 
Han, J., X. Weng and K. Bi. 2008. Antioxidants from a Chinese medicinal herb-Lithospermum erythrorhizon. Food Chem. 106:2-10.

Ignarro, L.J., M.L. Balestrieri and C. Napoli. 2007. Nutrition, physical activity, and cardiovascular disease: An update. Cardiovasc. Res. 73:326-340.

Lu, J., K. Ding and Q. Yuan. 2008. Determination of punicalagin isomers in pomegranate husk. Chromatographia 68:303-306.

Lu, J., K. Ding and Q. Yuan. 2011. One-step purification of punicalagin by preparative HPLC and stability study on punicalagin. Separ. Sci. Technol. 46:147-154.

Middha, S.K., T. Usha and V. Pande. 2013. HPLC evaluation of phenolic profile, nutritive content, and antioxidant capacity of extracts obtained from Punica granatum fruit peel. Adv. Pharmacol. Sci. 2013:1-6.

Negi, P.S., G.K. Jayaprakasha and B.S. Jena. 2003. Antioxidant and antimutagenic activities of pomegranate peel extracts. Food Chem. 80:393-397.

Noda, Y., T. Kaneyuka, A. Mori and L. Packer. 2002. Antioxidant activities of pomegranate fruit extract and its anthocyanidins; delphinidin, cyanidin, and pelargonidin. J. Agric. Food Chem. 50:166-171.

Orak, H.H., H. Yagar and S.S. Isbilir. 2012. Comparison of antioxidant activities of juice, peel, and seed of pomegranate (Punica granatum L.) and interrelationships with total phenolic, tannin, anthocyanin, and flavonoid contents. Food Sci. Biotechnol. 21:373387.
Reddy, M., S. Gupta, M. Jacob, S. Khan and D. Ferreira. 2007. Antioxidant, antimalarial and antimicrobial activities of tannin-rich fractions, ellagitannins and phenolic acids from Punica granatum L. Planta Med. 53:461-467.

Rusak, G., D. Komes, S. Likić, D. Horžić and M. Kovač. 2008. Phenolic content and antioxidative capacity of green and white tea extracts depending on extraction conditions and the solvent used. Food Chem. 110:852858.

Singh, R.P., K.N.C. Murthy and G.K. Jayaprakasha. 2002. Studies on the antioxidant activity of pomegranate peel and seed extracts using in vitro models. J. Agric. Food Chem. 50:81-86.

Singleton, V.L., R. Orthofer and R.M. Lamuela-Raventos. 1999. Analysis of total phenols and other oxidation substrates and antioxidants by means of folin-ciocalteu reagent. Methods Enzymol. 299:152-178.

Viuda-Martos, M., J. Fernandez-Lopez and J.A. PerezAlvarez. 2010. Pomegranate and its many functional components as related to human health: a review. Compr. Rev. Food Sci. Food Saf. 9:635-654.

Viuda-Martos, M., Y. Ruiz-Navajas, J. Fernández-López, E. Sendra, E. Sayas-Barberá and J.A. Pérez-Álvarez. 2011. Antioxidant properties of pomegranate (Punica granatum L.) bagasses obtained as co-product in the juice extraction. Food Res. Int. 44:1217-1223. 